\title{
Resposta de feijão-vagem a diferentes níveis de fertilidade
}

\author{
Nei Peixoto ${ }^{1}$; Leila T. Braz ${ }^{2}$; David A. Banzatto ${ }^{2}$; Ednan A. Moraes ${ }^{1}$; Francisco da M. Moreira ${ }^{3}$ \\ ${ }^{1}$ AGENCIARURAL, EE Anápolis, C. Postal 608, 75.001-970 Anápolis-GO, E-mail: nei.p@terra.com.br; ${ }^{2}$ UNESP-FCAV Depto. \\ Fitotecnia, 14.870-000 Jaboticabal-SP, E-mail: leilatb@fcav.unesp.br; ${ }^{3}$ UEG Anel Viário, 75.780-000 Ipameri-GO, E-mail: \\ sec.ipameri@ueg.rgi.com.br
}

\section{RESUMO}

Estudou-se a resposta de feijão-vagem de crescimento indeterminado à fertilidade do solo em Anápolis (GO). Utilizaramse sete linhagens (Hav 13, Hav 38, Hav 40, Hav 49, Hav 56, Hav 64 e Hav 68), e as cultivares Favorito Ag 480, Macarrão Bragança e Teresópolis Ag 484, em sete doses da formulação 4-30-16 aplicadas ao solo $\left(\mathrm{D}_{0}=0 ; \mathrm{D}_{1}=200 ; \mathrm{D}_{2} 400 ; \mathrm{D}_{3}=600 ; \mathrm{D}_{4}=800 ; \mathrm{D}_{5}=1000\right.$ e $\mathrm{D}_{6}=1200 \mathrm{~kg} / \mathrm{ha}$ ). Houve diferenças significativas entre genótipos para todas características avaliadas, dentro de cada dose do formulado, exceto para produtividade, no quinto nível, e para altura média das plantas no nível mais elevado de fertilidade. As linhagens Hav 49 e Hav 64 igualaram-se aos genótipos de maiores rendimentos em todos os ambientes. O nível médio de fertilidade foi o mais conveniente, do ponto de vista econômico, para todas as características consideradas. Com exceção de Hav 13 e Teresópolis Ag 484, que se mostraram imprevisíveis quanto à produtividade, Hav 13 e Hav 64, quanto ao número de vagens por planta e Favorito $\mathrm{Ag} 480$ quanto à altura média das plantas, os demais genótipos foram estáveis. Todos os genótipos responderam proporcionalmente à melhoria do ambiente para produtividade, número de vagens por planta e altura das plantas. Quanto ao peso médio das vagens a linhagem Hav 40 respondeu menos que proporcionalmente ao aumento da fertilidade do solo, mostrando-se adaptável a solos menos férteis, enquanto a cultivar Teresópolis Ag 484 respondeu mais que proporcionalmente à melhoria da fertilidade mostrando-se adaptável a solos mais férteis.

Palavras-chave: Phaseolus vulgaris, adaptabilidade, estabilidade.

\begin{abstract}
\section{Response of snap beans to soil fertility}

The response of snap beans to soil fertility was studied. Seven breeding lines (Hav 13, Hav 38, Hav 40, Hav 49, Hav 56, Hav 64 and Hav 68), and the cultivars Favorito Ag 480, Macarrão Bragança and Teresópolis Ag 484 were evaluated in seven NPK formulations (4-30-16), as basal fertilization $\left(\mathrm{D}_{0}=0 ; \mathrm{D}_{1}=200 ; \mathrm{D}_{2}=400 ; \mathrm{D}_{3}=600\right.$; $D_{4}=800 ; D_{5}=1,000$ and $\left.D_{6}=1,200 \mathrm{~kg} / \mathrm{ha}\right)$. There were significant differences among genotypes for all evaluated characteristics within each modified environment, except for yield, in the fifth level and for plant height in the highest fertility level. The breeding lines Hav 49 and Hav 64 produced as much as the higher yielding genotypes in all environments. The intermediate fertility level was the most advantageous under the economic viewpoint, for all considered characteristics. The genotypes were stable, except Hav 13 and Teresopolis Ag 484, unforeseeable in yield, Hav 13 and Hav 64, unforeseeable regarding to number of pods per plant and Favorito Ag 480, regarding to plant height. All genotypes presented a proportional response to environment improvement regarding to yield, number of pod per plant and plant height. The breeding line Hav 40 showed adaptability to lower fertility, while the cultivar Teresopolis Ag 484 showed to be adapted to higher fertility conditions, both of them regarding to pod medium weight.
\end{abstract}

Keywords: Phaseolus vulgaris, adaptability, stability.

\section{(Recebido para publicação em 14 de março de 2001 e aceito em 11 de outubro de 2002)}

$\mathrm{O}$ caracteres da morfologia do feijão-vagem podem ser classificados em constantes e variáveis. Os constantes determinam a taxonomia da espécie ou da cultivar, enquanto que os variáveis são resultantes da ação do meio sobre o genótipo e são influenciados pelo ambiente. Enquadram-se neste tipo os componentes de produção, adaptação e muitos aspectos de qualidade (Allard, 1960; Granval de Millan, 1990). A expressão fenotípica de caracteres variáveis depende da constituição genética, bem como do ambiente onde o genótipo é cultivado.

Um programa de melhoramento de feijão-vagem deve incluir ações como a identificação da amplitude da diversidade genética útil, relativas às características da planta e da vagem, resistên- cia a doenças e pragas e tolerância a estresses ambientais. Dentre os componentes ambientais destacam-se a temperatura, a umidade do solo e do ar, condições físico-químicas do solo, alta ou baixa temperatura durante $o$ florescimento, pegamento e desenvolvimento inicial das vagens e a capacidade de fixação simbiótica de $\mathrm{N}_{2}$ (Silbernagel, 1986). Esses fatores interagem, resultando na expressão fenotípica diferenciada dos genótipos, nos ambientes de cultivo.

Em geral estimam-se os efeitos dos componentes ambientais mais relevantes, tais como fertilidade do solo, pluviosidade e temperatura (Davis, 1945; Allard, 1960). Smith \& Pryor (1962), trabalhando com três cultivares de feijão, expostas a níveis crescentes de temperatura, verificaram redução na taxa de pegamento de vagens nos tratamentos com alta temperatura antes da polinização, mas as respostas foram diferenciadas, indicando diferenças genotípicas entre elas. Em feijão-delima, a alta temperatura noturna é favorável ao aparecimento de flores, mas reduz o pegamento das vagens, pois estimula a taxa respiratória, reduzindo-se os teores de amido e de açúcares; esta pode ser a causa da baixa capacidade de pegamento e retenção de vagens (Fischer \& Weaver, 1974).

Diversas metodologias visando estudar as interações genótipos $\mathrm{x}$ ambientes têm sido propostas, destacando-se aquelas que se baseiam nas análises de variância, na regressão linear e na regressão segmentada (Filgueira, 1991; 
Tabela 1. Produtividade (t/ha) de vagens comerciáveis de dez genótipos em sete microambientes. Anápolis, AGENCIARURAL, 1999.

\begin{tabular}{llllllll}
\hline \multirow{2}{*}{ Genótipo } & \multicolumn{5}{c}{ Microambientes $=$ doses } & (kg/ha) do formulado 04-30-16 no plantio \\
\cline { 2 - 8 } & \multicolumn{1}{c}{$\mathbf{0}$} & $\mathbf{2 0 0}$ & $\mathbf{4 0 0}$ & $\mathbf{6 0 0}$ & $\mathbf{8 0 0}$ & $\mathbf{1 0 0 0}$ & $\mathbf{1 2 0 0}$ \\
\hline Hav 13 & $17,8 \mathrm{ab} 1$ & $17,9 \mathrm{ab}$ & $19,3 \mathrm{ab}$ & $23,2 \mathrm{~cd}$ & $30,7 \mathrm{a}$ & $29,1 \mathrm{abc}$ & $34,6 \mathrm{a}$ \\
Hav 38 & $14,7 \mathrm{ab}$ & $19,7 \mathrm{a}$ & $20,9 \mathrm{ab}$ & $24,4 \mathrm{bcd}$ & $30,1 \mathrm{a}$ & $29,3 \mathrm{ab}$ & $27,7 \mathrm{ab}$ \\
Hav 40 & $15,5 \mathrm{ab}$ & $16,7 \mathrm{ab}$ & $19,2 \mathrm{ab}$ & $24,5 \mathrm{bcd}$ & $28,4 \mathrm{a}$ & $29,8 \mathrm{ab}$ & $28,4 \mathrm{ab}$ \\
Hav 49 & $13,3 \mathrm{ab}$ & $14,3 \mathrm{ab}$ & $18,4 \mathrm{ab}$ & $29,9 \mathrm{abc}$ & $27,5 \mathrm{a}$ & $31,2 \mathrm{ab}$ & $31,9 \mathrm{a}$ \\
Hav 56 & $15,3 \mathrm{ab}$ & $14,9 \mathrm{ab}$ & $17,8 \mathrm{ab}$ & $25,0 \mathrm{bcd}$ & $28,8 \mathrm{a}$ & $25,1 \mathrm{bc}$ & $27,1 \mathrm{ab}$ \\
Hav 64 & $20,0 \mathrm{a}$ & $17,6 \mathrm{ab}$ & $22,9 \mathrm{a}$ & $33,9 \mathrm{a}$ & $31,3 \mathrm{a}$ & $32,5 \mathrm{a}$ & $29,0 \mathrm{ab}$ \\
Hav 68 & $17,4 \mathrm{ab}$ & $17,5 \mathrm{ab}$ & $20,7 \mathrm{ab}$ & $26,2 \mathrm{bc}$ & $31,2 \mathrm{a}$ & 33,2 & $25,7 \mathrm{ab}$ \\
Favorito Ag 480 & $18,8 \mathrm{ab}$ & $13,6 \mathrm{ab}$ & $18,9 \mathrm{ab}$ & $25,9 \mathrm{bc}$ & $30,1 \mathrm{a}$ & $24,5 \mathrm{bc}$ & $27,1 \mathrm{ab}$ \\
Teresópolis Ag 484 & $16,4 \mathrm{ab}$ & $12,8 \mathrm{ab}$ & $14,4 \mathrm{~b}$ & $31,1 \mathrm{ab}$ & $28,1 \mathrm{a}$ & $27,0 \mathrm{abc}$ & $29,9 \mathrm{ab}$ \\
Macarrão Bragança & $11,2 \mathrm{~b}$ & $11,06 \mathrm{~b}$ & $12,8 \mathrm{~b}$ & $18,6 \mathrm{~d}$ & $24,0 \mathrm{a}$ & $22,1 \mathrm{c}$ & $20,9 \mathrm{~b}$ \\
\hline Média & $16,0 \mathrm{~B} 2$ & $15,6 \mathrm{~B}$ & $18,5 \mathrm{~B}$ & $26,3 \mathrm{~A}$ & $29,1 \mathrm{~A}$ & $28,4 \mathrm{~A}$ & $28,2 \mathrm{~A}$ \\
\hline CV (\%) & 15,0 & 19,09 & 15,27 & 9,05 & 11,82 & 8,46 & 13,2 \\
\hline
\end{tabular}

Médias seguidas da mesma letra minúscula, na coluna ${ }^{1}$, e maiúscula na linha ${ }^{2}$, não diferem entre si pelo teste de Tukey $(\mathrm{P}>0,05)$.

Gualberto, 1991; Cruz \& Regazzi, 1994). Atualmente as metodologias de Finlay \& Wilkinson (1963) e de Eberhart \& Russell (1966) têm sido as mais utilizadas, sendo ambas eficientes para descrever o comportamento dos genótipos frente às variações ambientais. A segunda, por utilizar escala aritmética, facilita a interpretação biológica dos resultados (Duarte 1988). Avaliou-se a adaptabilidade e a estabilidade de linhagens e cultivares de feijão-vagem de crescimento indeterminado em função de diferentes níveis de fertilidade do solo.

\section{MATERIAL E MÉTODOS}

Avaliaram-se, na EE de Anápolis, em solo de cerrado, plano e uniforme, dez genótipos em sete ambientes modificados (microambientes). Os genótipos constituíram-se de sete linhagens selecionadas, a partir de materiais introduzidos do Centro Internacional de Agricultura Tropical (Hav 13, Hav 64 e Hav 68, de vagens verdes, cilíndricas, Hav 38, Hav 49 e Hav 56 de vagens verde, achatadas e Hav 40 de vagens roxas, cilíndricas), três cultivares testemunhas: Favorito Ag 480 e Macarrão Bragança de vagens verde, cilíndricas e Teresópolis Ag 484, de vagem verde, achatadas. Os ambientes modificados (microambientes) foram obtidos por doses da formulação 4-30-16 no plan- tio $\left(\mathrm{D}_{0}=0 ; \mathrm{D}_{1}=200 ; \mathrm{D}_{2}=400 ; \mathrm{D}_{3}=600\right.$; $\mathrm{D}_{4}=800 ; \mathrm{D}_{5}=1000$ e $\left.\mathrm{D}_{6}=1200\right) \mathrm{kg} / \mathrm{ha}$.

Realizaram-se as adubações de plantio, de acordo com as doses previamente estabelecidas, incluindo-se como adubação comum $30 \mathrm{~m}^{3} /$ ha de esterco bovino. Em cobertura, utilizaram-se $300 \mathrm{~kg} /$ ha de sulfato de amônio divididos em duas aplicações, sendo um terço 20 dias após a semeadura e o restante 20 dias após a primeira.

Realizaram-se os tratos culturais e fitossanitários normais para a cultura, incluindo-se irrigação por aspersão convencional.

Obtiveram-se, por parcela, dados de número de plantas, peso e número de vagens comerciáveis, por colheita, sendo feitas duas colheitas por semana. Foram então calculados o número de vagens comerciáveis por planta, o peso médio de vagens e a produtividade. Os dados de altura média das plantas foram obtidos a partir de uma amostra de dez plantas por parcela, por ocasião da primeira colheita, medindo-se da base da planta ao seu ápice.

O delineamento experimental utilizado foi de blocos casualizados com três repetições, onde cada dose constituiu um experimento isolado, correspondente a cada um dos ambientes modificados. As parcelas tinham seis fileiras de plantas no de sistema tutorado com varas cruzadas, no espaçamento de $1,0 \mathrm{~m} \times 0,4$ $\mathrm{m}$, com duas plantas por cova. As par- celas úteis, com $4 \mathrm{~m}^{2}$, foram constituídas pelas duas fileiras centrais com 20 plantas.

Os dados foram submetidos à analise de interação genótipos $\mathrm{x}$ ambientes pelo método de Eberhart \& Russell (1966). Utilizou-se o aplicativo IGA, desenvolvido por Banzatto (1994), que realiza a análise conjunta dos dados dos diversos ambientes, segundo Yates \& Cochran (1938), a análise de variância e o teste de médias dentro de cada ambiente, segundo Banzatto \& Kronka (1989).

\section{RESULTADOS E DISCUSSÃO}

Foram significativas as interações genótipos $\mathrm{x}$ doses do formulado para todas as características, bem como as diferenças entre genótipos, dentro de cada ambiente, exceto para produtividade, na dose de $800 \mathrm{~kg} /$ ha e para altura média das planta na dose de $1200 \mathrm{~kg} / \mathrm{ha}$ (Tabelas 1, 2, 3 e 4).

As linhagens Hav 49 e Hav 64 igualaram-se em produtividade aos genótipos de maiores rendimentos em todos os ambientes. Nenhuma linhagem apresentou desempenho inferior às cultivares Favorito Ag 480 e Macarrão Bragança; a cultivar Teresópolis Ag 484 superou as linhagens Hav 13 e Hav 56 na dose de $600 \mathrm{~kg} /$ ha do formulado (Tabela 1).

A linhagem Hav 64 se igualou aos genótipos que produziram maior núme- 
Resposta de feijão-vagem a diferentes níveis de fertilidade

Tabela 2. Número de vagens comerciáveis por planta de dez genótipos em sete microambientes. Anápolis, AGENCIARURAL, 1999.

\begin{tabular}{|c|c|c|c|c|c|c|c|}
\hline \multirow{2}{*}{ Genótipo } & \multicolumn{7}{|c|}{ Microambientes $=$ doses $(\mathrm{kg} / \mathrm{ha})$ do formulado 04-30-16 no plantio } \\
\hline & 0 & 200 & 400 & 600 & 800 & 1000 & 1200 \\
\hline Hav 13 & $33,8 a b c^{1}$ & $37,8 a b$ & $39,5 a b c$ & $47,8 \mathrm{bc}$ & $66,1 \mathrm{ab}$ & $60,8 b c$ & $70,8 a$ \\
\hline Hav 38 & $34,8 a b c$ & $37,3 a b$ & $47,0 a b$ & $54,9 b c$ & $63,5 a b$ & $61,6 a b c$ & $62,8 \mathrm{abc}$ \\
\hline Hav 40 & $27,8 b c$ & $31,0 a b c$ & $38,2 \mathrm{bc}$ & $49,1 \mathrm{bc}$ & $57,0 \mathrm{abc}$ & $58,7 \mathrm{bcd}$ & $54,3 a b c d$ \\
\hline Hav 49 & $21,9 c$ & $21,3 b c$ & $28,9 \mathrm{~cd}$ & $44,5 b c$ & $41,2 \mathrm{e}$ & $45,8 \mathrm{de}$ & $48,2 \mathrm{~cd}$ \\
\hline Hav 56 & $37,1 \mathrm{abc}$ & $35,6 a b c$ & $44,3 a b c$ & $59,2 \mathrm{bc}$ & $64,7 a b$ & $56,1 \mathrm{~cd}$ & $63,6 a b c$ \\
\hline Hav 64 & $51,4 a$ & $44,1 \mathrm{a}$ & $55,1 \mathrm{a}$ & $83,2 a$ & $75,0 a$ & $76,3 a$ & $67,1 \mathrm{ab}$ \\
\hline Hav 68 & $39,7 a b c$ & $39,4 a b$ & $47,9 a b$ & $60,4 b$ & $68,1 a b$ & $72,1 \mathrm{ab}$ & $56,0 a b c d$ \\
\hline Favorito Ag 480 & $45,0 a b$ & $32,3 a b c$ & $43,8 a b c$ & $57,3 b c$ & $62,7 a b$ & $55,0 \mathrm{~cd}$ & $57,5 a b c$ \\
\hline Teresópolis Ag 484 & $24,0 \mathrm{c}$ & $15,7 c$ & $20,3 d$ & $42,7 \mathrm{bc}$ & $38,1 \mathrm{c}$ & $35,3 e$ & $38,5 d$ \\
\hline Macarrão Bragança & $28,6 b c$ & $26,3 a b c$ & $32,0 \mathrm{bcd}$ & $38,2 \mathrm{c}$ & $55,0 b c$ & $51,6 \mathrm{~cd}$ & $51,3 \mathrm{bcd}$ \\
\hline Média & $34,4 \mathrm{BC}^{2}$ & $32,1 \mathrm{C}$ & $39,7 \mathrm{~B}$ & $53,7 \mathrm{~A}$ & $59,2 \mathrm{~A}$ & $57,3 \mathrm{~A}$ & $57,0 \mathrm{~A}$ \\
\hline$\overline{C V}(\%)$ & 17,9 & 14,1 & 14,1 & 13,7 & 11,5 & 8,8 & 11,1 \\
\hline
\end{tabular}

Médias seguidas da mesma letra minúscula, na coluna ${ }^{1}$, e maiúscula na linha ${ }^{2}$, não diferem entre si pelo teste de Tukey $(\mathrm{P}>0,05)$.

Tabela 3. Peso médio (g) de vagens comerciáveis de dez genótipos em sete microambientes. Anápolis, AGENCIARURAL, 1999.

\begin{tabular}{lccccccc}
\hline \multirow{2}{*}{ Genótipo } & \multicolumn{7}{c}{ Microambientes $=$ doses $\mathbf{( k g / h a ) ~ d o ~ f o r m u l a d o ~ 0 4 - 3 0 - 1 6 ~ n o ~ p l a n t i o ~}$} \\
\cline { 2 - 8 } & $\mathbf{0}$ & $\mathbf{2 0 0}$ & $\mathbf{4 0 0}$ & $\mathbf{6 0 0}$ & $\mathbf{8 0 0}$ & $\mathbf{1 0 0 0}$ & $\mathbf{1 2 0 0}$ \\
\hline Hav 13 & $9,2 \mathrm{~b}^{1}$ & $9,4 \mathrm{bc}$ & $9,8 \mathrm{~cd}$ & $9,7 \mathrm{bc}$ & $9,3 \mathrm{bc}$ & $9,6 \mathrm{~cd}$ & $9,8 \mathrm{~cd}$ \\
Hav 38 & $8,5 \mathrm{~b}$ & $8,5 \mathrm{c}$ & $8,9 \mathrm{cde}$ & $9,1 \mathrm{bc}$ & $9,5 \mathrm{bc}$ & $9,6 \mathrm{~cd}$ & $8,8 \mathrm{~cd}$ \\
Hav 40 & $11,6 \mathrm{a}$ & $10,7 \mathrm{~b}$ & $10,0 \mathrm{c}$ & $10,2 \mathrm{~b}$ & $10,0 \mathrm{~b}$ & $10,2 \mathrm{c}$ & $10,5 \mathrm{c}$ \\
Hav 49 & $12,0 \mathrm{a}$ & $13,3 \mathrm{a}$ & $12,7 \mathrm{~b}$ & $13,5 \mathrm{a}$ & $13,3 \mathrm{a}$ & $13,6 \mathrm{~b}$ & $13,3 \mathrm{~b}$ \\
Hav 56 & $8,1 \mathrm{~b}$ & $8,4 \mathrm{c}$ & $8,1 \mathrm{e}$ & $8,4 \mathrm{c}$ & $8,9 \mathrm{bc}$ & $9,0 \mathrm{~cd}$ & $8,3 \mathrm{~d}$ \\
Hav 64 & $7,8 \mathrm{~b}$ & $7,9 \mathrm{c}$ & $8,3 \mathrm{e}$ & $8,1 \mathrm{c}$ & $8,4 \mathrm{c}$ & $8,5 \mathrm{~d}$ & $9,0 \mathrm{~cd}$ \\
Hav 68 & $8,8 \mathrm{~b}$ & $8,9 \mathrm{c}$ & $8,7 \mathrm{cde}$ & $8,7 \mathrm{bc}$ & $9,2 \mathrm{bc}$ & $9,2 \mathrm{~cd}$ & $9,3 \mathrm{~cd}$ \\
Favorito Ag 480 & $8,3 \mathrm{~b}$ & $8,5 \mathrm{c}$ & $8,6 \mathrm{de}$ & $9,0 \mathrm{bc}$ & $9,7 \mathrm{bc}$ & $9,0 \mathrm{~cd}$ & $9,2 \mathrm{~cd}$ \\
Teresópolis Ag 484 & $13,7 \mathrm{a}$ & $14,1 \mathrm{a}$ & $14,3 \mathrm{a}$ & $14,6 \mathrm{a}$ & $14,7 \mathrm{a}$ & $15,3 \mathrm{a}$ & $15,6 \mathrm{a}$ \\
Macarrão Bragança & $7,8 \mathrm{~b}$ & $8,2 \mathrm{c}$ & $8,0 \mathrm{e}$ & $8,5 \mathrm{bc}$ & $8,7 \mathrm{bc}$ & $8,6 \mathrm{~d}$ & $8,1 \mathrm{~d}$ \\
\hline Média & $9,6 \mathrm{~B}^{2}$ & $9,8 \mathrm{AB}$ & $9,7 \mathrm{AB}$ & $10,0 \mathrm{~A}$ & $10,2 \mathrm{~A}$ & $10,3 \mathrm{~A}$ & $10,2 \mathrm{~A}$ \\
\hline CV (\%) & 8,4 & 6,0 & 5,0 & 6,12 & 4,8 & 4,2 & 5,7 \\
\hline
\end{tabular}

Médias seguidas da mesma letra minúscula, na coluna ${ }^{1}$, e maiúscula na linha ${ }^{2}$, não diferem entre si pelo teste de Tukey (P>0,05).

ro de vagens por planta. Superou as cultivares testemunhas Macarrão Bragança e Teresópolis Ag 484 em todos ambientes e Favorito Ag 480 nas doses de 600 e $1000 \mathrm{~kg} /$ ha do formulado (Tabela 2).

As quatro maiores doses do formulado resultaram em vagens com maior peso médio do que a testemunha com dose zero. Os maiores pesos médios foram da cultivar Teresópolis Ag 484, seguida de Hav 49, ambas com vagens achatadas e a menor média pela linhagem Hav 64 (Tabela 3).

A linhagem Hav 64 e as cultivares Favorito Ag 480 e Macarrão Bragança, tenderam a apresentar porte mais baixo, independente das doses do formulado. A altura média das plantas foi crescente até $600 \mathrm{~kg} /$ ha do formulado, estabilizando-se a partir desta (Tabela 4).

A dose média do formulado $(600 \mathrm{~kg} /$ ha), foi a mais conveniente, do ponto de vista econômico. As três menores doses caracterizaram os ambientes desfavoráveis e as quatro maiores os ambientes favoráveis (Tabelas 1, 2, 3, 4).

Os genótipos responderam proporcionalmente à melhoria do ambiente, $\left(b_{i}=1\right)$, para número de vagens por planta e altura das plantas. A linhagem Hav 40 respondeu, quanto ao peso médio das vagens, menos que proporcionalmente $\left(b_{i}<1\right)$ pela melhoria do ambiente, mostrando-se adaptável a ambientes de menor fertilidade, ao contrário de Teresópolis Ag 484, adaptável a ambientes de maior fertilidade $\left(b_{i}>1\right)$ para esta mesma característica. Com exceção de Hav 13 e Teresópolis Ag 484, que se mostraram imprevisíveis $\left(\mathrm{s}^{2} \mathrm{~d}_{\mathrm{i}}\right.$ ?0) quanto à produtividade, Hav 13 e Hav 64, quanto ao número de vagens por planta e Favorito Ag 480, quanto à altura média das plantas, os demais genótipos foram estáveis $\left(\mathrm{s}^{2} \mathrm{~d}_{\mathrm{i}}=0\right)$. 
Tabela 4. Altura média das plantas de dez genótipos em sete microambientes. Anápolis, AGENCIARURAL, 1999.

\begin{tabular}{|c|c|c|c|c|c|c|c|}
\hline \multirow{2}{*}{ Genótipo } & \multicolumn{7}{|c|}{ Microambientes $=$ doses $(\mathrm{kg} / \mathrm{ha})$ do formulado $04-30-16$ no plantio } \\
\hline & 0 & 200 & 400 & 600 & 800 & 1000 & 1200 \\
\hline Hav 13 & $139,9 a b^{1}$ & $199,5 a b$ & $208,3 a b$ & $229,2 a b$ & $225,7 a b c$ & $215,9 b c$ & $221,8 a$ \\
\hline Hav 38 & $158,8 a$ & $179,5 a b$ & $219,2 a b$ & $235,3 a$ & $221,3 a b c$ & $230,5 a b$ & $222,7 a$ \\
\hline Hav 40 & $155,8 a$ & $210,0 a$ & $222,9 a$ & $220,7 a b c$ & $236,0 a$ & $237,9 a$ & $230,7 a$ \\
\hline Hav 49 & $155,8 a$ & $205,7 a$ & $227,0 a$ & $227,4 a b$ & $228,9 a b$ & $238,5 a$ & $236,3 a$ \\
\hline Hav 56 & $137,6 a b$ & $184,9 a b$ & $202,1 \mathrm{ab}$ & $207,8 \mathrm{abcd}$ & $211,0 a b c d$ & $233,7 a b$ & $226,9 a$ \\
\hline Hav 64 & $109,9 b$ & $168,2 \mathrm{ab}$ & $196,2 \mathrm{ab}$ & $196,7 \mathrm{~cd}$ & $182,3 d$ & $216,3 b c$ & $202,9 a$ \\
\hline Hav 68 & $154,3 a$ & $180,8 a b$ & $203,0 a b$ & $215,5 \mathrm{abcd}$ & $226,3 a b c$ & $233,1 \mathrm{ab}$ & $220,8 a$ \\
\hline Favorito $\mathrm{Ag} 480$ & $118,7 a b$ & $138,4 b$ & $147,3 \mathrm{c}$ & $203,1 \mathrm{~cd}$ & $203,9 \mathrm{bcd}$ & $199,9 c$ & $209,1 a$ \\
\hline Teresópolis Ag 484 & $140,6 a b$ & $182,1 \mathrm{ab}$ & $199,5 a b$ & $208,3 a b c d$ & $226,3 a b c$ & $234,0 a b$ & $228,3 a$ \\
\hline Macarrão Bragança & $119,7 a b$ & $153,7 a b$ & $172,6 \mathrm{bc}$ & $188,7 d$ & $196,1 \mathrm{~cd}$ & $197,7 \mathrm{c}$ & $203,0 a$ \\
\hline Média & $139,1 D^{2}$ & $180,3 \mathrm{C}$ & 199,8B & $213,3 \mathrm{~A}$ & $215,79 \mathrm{~A}$ & $223,8 \mathrm{~A}$ & $220,3 \mathrm{~A}$ \\
\hline CV (\%) & 10,7 & 12,1 & 8,3 & 4,9 & 4,9 & 3,3 & 6,0 \\
\hline
\end{tabular}

Médias seguidas da mesma letra minúscula, na coluna ${ }^{1}$, e maiúscula na linha ${ }^{2}$, não diferem entre si pelo teste de Tukey $(\mathrm{P}>0,05)$.

\section{LITERATURA CITADA}

ALLARD, R.W. Principles of plant breeding. New York: Willey., 1960, 485 p.

BANZATTO, D.A. Comparação de métodos de avaliação da adaptabilidade e estabilidade de cultivares de batata. Jaboticabal: FCAV, 1994. 170 p. (Tese livre docência).

BANZATTO, D.A.; KRONKA, S.N. Experimentação Agrícola. Jaboticabal: FUNEP, 1989. 247 p CRUZ, C.D.; REGAZZI, A.J. Modelos biométricos aplicados ao melhoramento de plantas. Viçosa. UFV, Imprensa Universitária, 1994. $330 \mathrm{p}$.

DAVIS, J.F. The effects of some environmental factors on the set of pods and yield of cowpea beans. Journal of Agricultural Research, v. 70, n. 7. p. $237-249,1945$.
DUARTE, J.B. Estudo da adaptabilidade e estabilidade fenotipica em linhagens e cultivares de feijão mulatinho (Phaseolus vulgaris L.). Goiânia, 1988, 155 p. (Tese mestrado)

EBERHART, S.A.; RUSSELL, W.A. Stability parameters for comparing varieties. Crop Science, v. 6, p. $36-40,1966$.

FILGUEIRA, F.A.R. Interação genótipo $x$ ambiente em batata (Solanum tuberosum L. ssp.tuberosum). Jaboticabal: FCAV, 1991. 121 p. (Tese doutorado)

FINLAY, K.W.; WILKINSON, G.N. The analysis of adaptation in a plant breeding programme. Australian Journal of Agricultural Research, Melbourne, v. 14, n. 6, p. 742-754, 1963.

FISCHER, V.J.; WEAVER, C.K. Flowering, pod set and retention of lima bean in response to high temperature, humidity and soil moisture. Journal American Society Horticultural Science, v. 99, n. 5, p. 448-450, 1974.
GUALBERTO, R. Análise da estabilidade fenotípica de cultivares de batata (Solanum tuberosum L.) na região sul de Minas Gerais. Lavras, 1991, 101 p. (Tese mestrado)

GRANVAL DE MILLAN, N.I. Aspectos practicos del mejoramiento y la producción de semilla de poroto chaucha. In: CURSO/TALLER EN TECNOLOGIA DE PRODUCCIÓN DE SEMILLAS HORTICOLAS PARA PEQUEÑOS AGRICULTORES. Mendoza, INTA/FAO. 1990, p. 205-226.

SILBERNAGEL, M.J. Snap bean breeding. In.:BASSETT, M.J. (Coord.). Breeding vegetable crops. Westport, Avi. Publ. Comp., 1986. p. 243282

SMITH, F.L; PRYOR, R.H. Effects of maximum temperature and age of flowering and seed production in three bean varieties. Hilgardia. v. 33, n. 12, p. 669-688, 1962.

YATES, F.; COCHRAN, W.G. The analysis of groups of experiments. The Journal of Agricultural Science, v. 28, n. 4, p. 556-580,1938. 